1-20-1995

\title{
Improved Gaussian Beam-Scattering Algorithm
}

James A. Lock

Cleveland State University, j.lock@csuohio.edu

Follow this and additional works at: https://engagedscholarship.csuohio.edu/sciphysics_facpub

Part of the Physics Commons

How does access to this work benefit you? Let us know!

\section{Publisher's Statement}

This paper was published in Applied Optics and is made available as an electronic reprint with the permission of OSA. The paper can be found at the following URL on the OSA website: http://www.opticsinfobase.org/ao/abstract.cfm?URI=ao-34-3-559. Systematic or multiple reproduction or distribution to multiple locations via electronic or other means is prohibited and is subject to penalties under law.

\section{Original Citation}

Lock, James A. "Improved Gaussian Beam-Scattering Algorithm." Applied Optics 34 (1995): 559-570.

\section{Repository Citation}

Lock, James A., "Improved Gaussian Beam-Scattering Algorithm" (1995). Physics Faculty Publications. 40.

https://engagedscholarship.csuohio.edu/sciphysics_facpub/40

This Article is brought to you for free and open access by the Physics Department at EngagedScholarship@CSU. It has been accepted for inclusion in Physics Faculty Publications by an authorized administrator of

EngagedScholarship@CSU. For more information, please contact library.es@csuohio.edu. 


\title{
Improved Gaussian beam-scattering algorithm
}

\author{
James A. Lock
}

\begin{abstract}
The localized model of the beam-shape coefficients for Gaussian beam-scattering theory by a spherical particle provides a great simplification in the numerical implementation of the theory. We derive an alternative form for the localized coefficients that is more convenient for computer computations and that provides physical insight into the details of the scattering process. We construct a FORTRAN program for Gaussian beam scattering with the localized model and compare its computer run time on a personal computer with that of a traditional Mie scattering program and with three other published methods for computing Gaussian beam scattering. We show that the analytical form of the beam-shape coefficients makes evident the fact that the excitation rate of morphology-dependent resonances is greatly enhanced for far off-axis incidence of the Gaussian beam.
\end{abstract}

\section{Introduction}

Although the history of numerical Mie theory computations dates back almost to the time of Mie and Debye, ${ }^{1}$ it was not until a widely published numerically stable computer code ${ }^{2-4}$ could be run quickly on low-cost personal computers ${ }^{5}$ that plane-wave Mie theory computations became commonplace. In recent years progress has al so been made on thenumerical implementation of other scattering problems. For example, a number of computational methods for calculation of scattering of a focused Gaussian beam by a spherical particle have been devised. In these methods the Gaussian beam is expanded either in an infinite series of particle waves ${ }^{6-8}$ or in an angular spectrum of plane waves. ${ }^{9-11}$ These expansions have considered not only the Gaussian shape of the dominant component of the beam's electric and magnetic fields, but also included in an approximate way smaller components of the fields induced by the variation of the dominant component in the transverse direction. ${ }^{12,13}$

Currently no consensus has been reached as to which computational method for Gaussian beam scattering is superior to the others or whether one method possesses a richness of physical interpretation that is not manifest in the others. This is because the successes and limitations of each method have not yet been fully explored. To this end, in two recent pa-

The author is with the Department of Physics, Cleveland State University, Cleveland, Ohio 44115.

Received 25 March 1994; revised manuscript received 13 J uly 1994.

0003-6935/95/030559-12\$06.00/0.

(c) 1995 Optical Society of America. pers ${ }^{14,15}$ we examined the applicability of Gouesbet's localized model ${ }^{16,17}$ of Gaussian beam scattering to the case of tight beam local ization. We found that for an on-axis beam, i.e., a beam propagating along the $z$ axis, the localized model accurately describes a focused Gaussian beam with the focal waist half-width $\mathrm{w}_{0}$ satisfying $\lambda /\left(2 \pi \mathrm{w}_{0}\right) \lesssim 0.15$. For an off-axis beam, i.e., a beam propagating parallel to but not al ong the $z$ axis, thelocalized model accurately describes a Gaussian beam for $\lambda /\left(2 \pi w_{0}\right) \lesssim 0.10$, although the accuracy depends to some extent on the aspect of the scattering that is being examined. In this paper we consider a different application of the localized model, namely, the construction of a stable and relatively fastrunning computer code for Gaussian beam scattering that can be implemented on a personal computer.

The body of this paper proceeds as follows. In Section 2 we give the basic formulas for far-field scattering of a focused off-axis Gaussian beam by a spherical particle. We also give the localized model expressions for the beam-shape coefficients that describe the Gaussian beam. We then simplify the localized expressions, writing them in terms of either Bessel functions or modified Bessel functions of a complex argument. In Section 3 we describe algorithms for numerical computation of the Bessel functions and other expressions that appear in the farfield scattering formulas. In Section 4 we examine the computer run time of our Gaussian beamscattering program and compare it with the run time of a standard plane-wave Mie theory program. We also compare our program with three other computational methods for Gaussian beam scattering. Last, in Section 5 we show that the analytical expressions 
for the beam-shape coefficients easily and correctly predict the large enhancement in the excitation rate of morphology-dependent resonances (MDR's) by an off-axis Gaussian beam focused somewhat beyond the edge of a dielectric spherical particle.
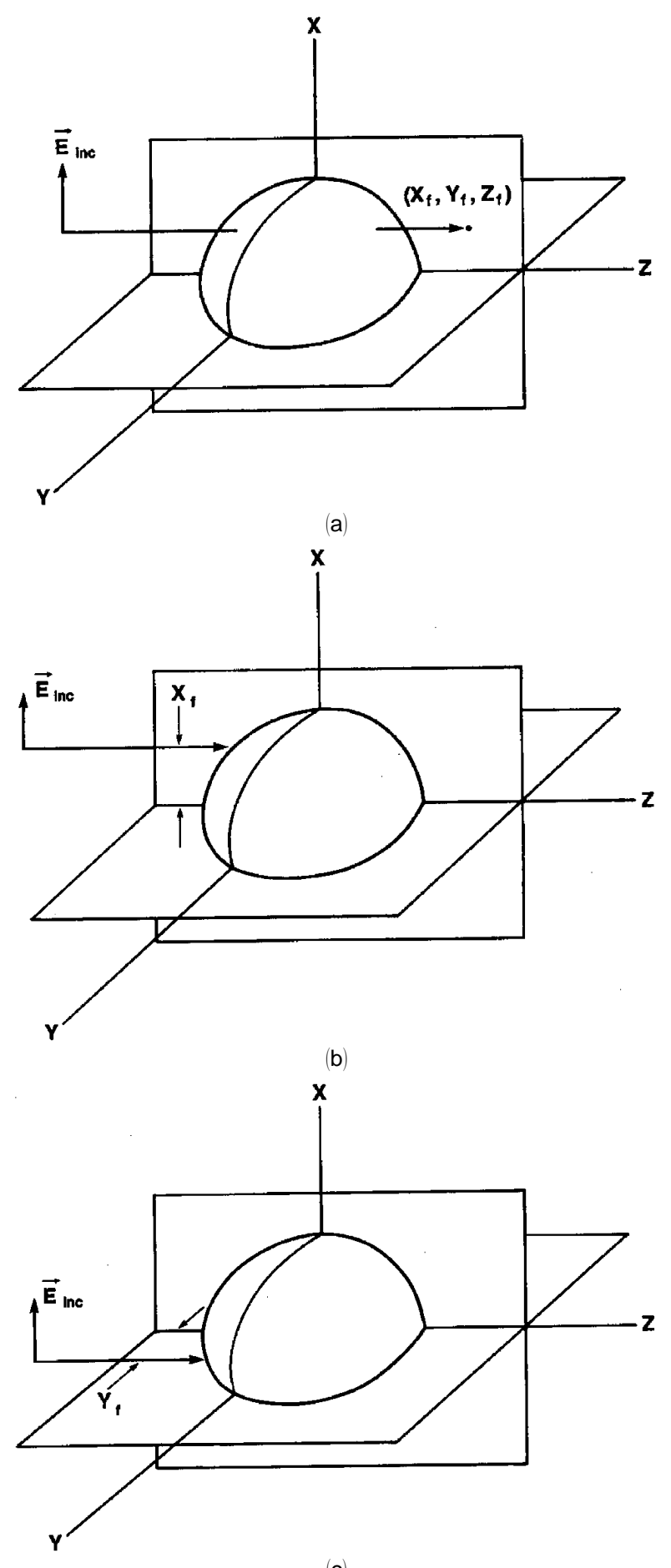

(c)

Fig. 1. Focused Gaussian beam that is incident upon a spherical particle. The center of the particle is at the origin of the coordinates, and the center of the beam's focal waist is at $(a) x_{f}, y_{f}, z_{f}$; (b) $x_{f} \neq 0, y_{f}=0$; and $(c) x_{f}=0, y_{f} \neq 0$.

\section{Localized Model of Gaussian Beam Scattering}

Consider a Gaussian beam focused to the half-width $\mathrm{w}_{0}$ at the point $\left(\mathrm{x}_{\mathrm{f}}, \mathrm{y}_{\mathrm{f}} \mathrm{Z}_{\mathrm{f}}\right)$ that is incident upon $\mathrm{a}$ spherical particle whose center is at the origin of coordinates. This is shown in Fig. 1(a). The radial components of the beam's el ectric and magnetic fields are $E_{\text {inc }}{ }^{\text {rad }}(r, \theta, \phi)$ and $B_{\text {inc }}{ }^{\text {rad }}(r, \theta, \phi)$, respectively. The spherical particle has radius a and refractive index $\mathrm{n}$. In the notation of Ref. 18, the far-field scattered intensity is

$$
\mathrm{I}(\mathrm{r}, \theta, \phi)=\frac{1}{2 \mu_{0} \mathrm{ck}^{2} \mathrm{r}^{2}}\left[\left|\mathrm{~S}_{1}(\theta, \phi)\right|^{2}+\left|\mathrm{S}_{2}(\theta, \phi)\right|^{2}\right],
$$

where the scattering amplitudes $S_{1}$ and $S_{2}$ are given by

$$
\begin{aligned}
\mathrm{S}_{1}(\theta, \phi)= & \sum_{\mathrm{l}=1}^{\infty} \sum_{\mathrm{m}=-1}^{\mathrm{l}} \frac{2 \mathrm{l}+1}{2 \mathrm{l}(\mathrm{I}+1)}\left[-\mathrm{im} \alpha_{\mathrm{Im}} \pi_{\mid} \mid \mathrm{m} /(\theta)\right. \\
& \left.+\beta_{\mid \mathrm{m}} \tau_{l}|\mathrm{~m}|(\theta)\right] \exp (\mathrm{im} \phi), \\
\mathrm{S}_{2}(\theta, \phi)= & \sum_{\mathrm{l}=1}^{\infty} \sum_{\mathrm{m}=-1}^{\mathrm{I}} \frac{2 \mathrm{l}+1}{2 \mathrm{l}(\mathrm{I}+1)}\left[\mathrm{im} \beta_{\mid \mathrm{m}} \pi_{\mid}|\mathrm{m}|(\theta)\right. \\
& \left.+\alpha_{\mid \mathrm{m}} \tau_{l}|\mathrm{~m}|(\theta)\right] \exp (\mathrm{im} \phi) .
\end{aligned}
$$

In Eqs. (1) and (2)

$$
k=\frac{2 \pi}{\lambda}
$$

is the wave number of the incident beam. The partial-wave scattering amplitudes $\alpha_{I m}$ and $\beta_{I m}$ are given by

$$
\begin{aligned}
& \alpha_{I m}=A_{I m} a_{1}, \\
& \beta_{I m}=B_{I m} b_{\mid},
\end{aligned}
$$

respectively, where $a_{1}$ and $b_{1}$ are the partial-wave scattering amplitudes of plane-wave Mie theory. Efficient algorithms for the computation of $a_{1}$ and $b_{1}$ are given in Refs. 3 and 4 . The beam-shape coefficients $A_{I m}$ and $B_{I m}$ are

$$
\begin{aligned}
A_{I m}= & \frac{(-i)^{I-1}}{2 \pi} \frac{k r}{j_{I}(k r)} \frac{(I-|m|) !}{(I+|m|) !} \int_{0}^{\pi} \sin \theta d \theta \\
& \times \int_{0}^{2 \pi} d \phi P_{\mid} \mid m /(\cos \theta) \exp (-i m \phi) E_{i n c}{ }^{r a d}(r, \theta, \phi), \\
B_{I m}= & \frac{(-i)^{l-1}}{2 \pi} \frac{k r}{j_{I}(k r)} \frac{(I-|m|) !}{(I+|m|) !} \int_{0}^{\pi} \sin \theta d \theta \\
& \times \int_{0}^{2 \pi} d \phi P_{I}|m|(\cos \theta) \exp (-i m \phi) B_{\text {inc }}^{r a d}(r, \theta, \phi),
\end{aligned}
$$


respectively, where $\mathrm{j}_{1}(\mathrm{kr})$ are spherical Bessel functions. The angular functions $\pi_{\mid}{ }^{\mathrm{m}} \mathrm{m}(\theta)$ and $\tau_{\mid} \mathrm{m}(\theta)$ are given by

$$
\begin{aligned}
\pi_{\mid} \mid \mathrm{m}(\theta) & =\frac{1}{\sin \theta} \mathrm{P}_{\mid}|\mathrm{m}|(\cos \theta), \\
\tau_{\mid} \mathrm{m}(\theta) & =\frac{\mathrm{d}}{\mathrm{d} \theta} \mathrm{P}_{\mid}|\mathrm{m}|(\cos \theta),
\end{aligned}
$$

respectively, where $P_{\mid} \mid m(\cos \theta)$ are associated Legendre polynomials.

Calculation of the beam-shape coefficients of Eqs. (5) by the use of numerical integration is computationally the most time-consuming task in the numerical implementation of Eqs. (1)-(6). This is because the integrands are rapidly varying functions of $\theta$ and $\phi$, i.e., because of the $P_{1} m(\cos \theta)$ and $\exp (-i m \phi)$ factors and the fact that $\mathrm{E}_{\text {inc }}{ }^{\text {rad }}$ and $\mathrm{B}_{\text {inc }}{ }^{\text {rad }}$ are proportional to $\exp (i k r \cos \theta)$ with the usual evaluation criterion $r=$ a. Thus dense grids are required in both the $\theta$ and the $\phi$ directions to obtain convergence of the numerical integrations. ${ }^{18}$ The localized model approximates the integrals in Eqs. (5) with an analytical function, thus decreasing the computer run time many fold. For an off-axis-focused Gaussian beam, the localized model of the beam-shape coefficients is ${ }^{17}$

$$
\begin{aligned}
& A_{I m}{ }^{l o c}=\frac{K_{I m}}{2} F_{I} \sum_{j=0}^{\infty} \sum_{p=0}^{j}\left(\bar{\Psi}_{j p} \delta_{j-2 p+1, m}+\bar{\Psi}_{j p} \delta_{j-2 p-1, m}\right), \\
& B_{I m}{ }^{l o c}=\frac{K_{I m}}{2 i} F_{\mid} \sum_{j=0}^{\infty} \sum_{p=0}^{j}\left(\bar{\Psi}_{j p} \delta_{j-2 p+1, m}-\bar{\Psi}_{j p} \delta_{j-2 p-1, m}\right),
\end{aligned}
$$

where

$$
\begin{aligned}
& F_{1}=D \exp \left[-D\left(\frac{x_{f}^{2}+y_{f}^{2}}{w_{0}^{2}}\right)\right] \exp \left[-D s^{2}\left(I+\frac{1}{2}\right)^{2}\right] \\
& \times \exp \left(\frac{-i}{s} \frac{z_{f}}{w_{0}}\right) \text {, } \\
& \mathrm{s}=\frac{1}{\mathrm{kw}_{0}}, \\
& D=\left(1-\frac{2 i s z_{f}}{w_{0}}\right)^{-1}, \\
& K_{I m}= \begin{cases}\frac{2 i l(I+1)}{I+\frac{1}{2}} & \text { if } m=0 \\
2\left(\frac{-i}{I+\frac{1}{2}}\right)^{|m|-1} & \text { if } m \neq 0,\end{cases} \\
& \bar{\Psi}_{j p}=\left[s\left(I+\frac{1}{2}\right) D\right]^{j} \frac{\left(\frac{x_{f}-i y_{f}}{w_{0}}\right)^{j-p}}{(j-p) !} \frac{\left(\frac{x_{f}+i y_{f}}{w_{0}}\right)^{p}}{p !} .
\end{aligned}
$$

The accuracy of Eqs. (7)-(12) in approximating Eqs. (5) for a focused off-axis Gaussian beam was examined in Refs. 15 and 17-19. Equations (7)-(12), however, are not in optimal form for numerical computations. In particular the Kronecker delta implies that for a given value of $j$, only one value of $p$ is considered. When this is taken into account, the resulting infinite series in $\mathrm{j}$ is recognized as that of a Bessel function. ${ }^{20}$ The localized beam-shape coefficients $A_{I m}{ }^{\text {loc }}$ and $B_{I m}{ }^{\text {loc }}$ may then be written in the more compact form

$$
A_{I m}{ }^{l o c}= \begin{cases}A_{I m}{ }^{+l o c} & \text { if } m>0 \\ A_{I m}{ }^{-l o c} & \text { if } m<0 \\ A_{I 0}{ }^{l o c} & \text { if } m=0,\end{cases}
$$

where

$$
\begin{aligned}
& A_{I m} \pm 10 c=F_{1}\left(\frac{-r_{f}^{\mp}}{I+\frac{1}{2}}\right)^{m-1}\left[J_{m-1}(P)-\left(r_{f}^{\mp}\right)^{2} J_{m+1}(P)\right], \\
& A_{I 0} 10 c=F_{I} \frac{2 I\left(I+\frac{1}{1}\right)}{\left(I+\frac{1}{2}\right)} \frac{x_{f}}{\left(x_{f}^{2}+y_{f}^{2}\right)^{1 / 2}} J_{1}(P),
\end{aligned}
$$

with

$$
\begin{aligned}
r_{f}^{ \pm} & =\frac{x_{f} \pm i y_{f}}{\left(x_{f}^{2}+y_{f}^{2}\right)^{1 / 2}}, \\
P & =\frac{-\left(I+\frac{1}{2}\right)}{z_{f} / w_{0}}\left(\frac{x_{f}^{2}+y_{f}^{2}}{w_{0}^{2}}\right)^{1 / 2}\left(1+\frac{i w_{0}}{2 s z_{f}}\right)^{-1} .
\end{aligned}
$$

Similarly,

$$
B_{I m}{ }^{l o c}= \begin{cases}B_{I m}{ }^{+l o c} & \text { if } m>0 \\ B_{I m}{ }^{-l o c} & \text { if } m<0 \\ B_{10}{ }^{l o c} & \text { if } m=0\end{cases}
$$

where

$$
\begin{aligned}
B_{I m} \pm l o c & =\frac{ \pm F_{1}}{i}\left(\frac{-r_{f}^{\mp}}{I+\frac{1}{2}}\right)^{m-1}\left[J_{m-1}(P)+\left(r_{f}^{\mp}\right)^{2} J_{m+1}(P)\right], \\
B_{10}{ }^{l o c} & =F_{1} \frac{2 l\left(I+\frac{1}{1}\right)}{\left(I+\frac{1}{2}\right)} \frac{y_{f}}{\left(x_{f}^{2}+y_{f}^{2}\right)^{1 / 2}} J_{1}(P),
\end{aligned}
$$

Equations (14)-(18) simplify further when the beam waist and the particle orientation are such that $x_{f} \neq 0$, $y_{f}=0$ or when $x_{f}=0, y_{f} \neq 0$ [see Figs. $1(b)$ and $1(c)$ ]. If $x_{f}=y_{f}=0$, the coefficients reduce to their values in the on-axis localized model. ${ }^{16}$ 
Because Bessel functions and modified Bessel functions are related to each other by ${ }^{21}$

$$
J_{n}(x)=i^{n}{ }_{n}(-i x),
$$

Eqs. (14)-(18) may be rewritten as

$$
\begin{aligned}
& A_{I m} \pm l o c=F_{1}\left(\frac{-i r_{f}^{\mp}}{I+\frac{1}{2}}\right)^{m-1}\left[I_{m-1}(Q)+\left(r_{f}^{\mp}\right)^{2} I_{m+1}(Q)\right], \\
& A_{10}{ }^{\circ o c}=F_{1} \frac{2 i l(I+1)}{\left(I+\frac{1}{2}\right)} \frac{x_{f}}{\left(x_{f}^{2}+y_{f}^{2}\right)^{1 / 2}} l_{1}(Q), \\
& \mathrm{B}_{\mathrm{Im}} \pm \mathrm{loc}=\frac{ \pm \mathrm{F}_{1}}{\mathrm{i}}\left(\frac{-\mathrm{ir}_{\mathrm{f}}^{\mp}}{\mathrm{I}+\frac{1}{2}}\right)^{\mathrm{m}-1}\left[\mathrm{I}_{\mathrm{m}-1}(\mathrm{Q})-\left(\mathrm{r}_{\mathrm{f}}^{\mp}\right)^{2} \mathrm{I}_{\mathrm{m}+1}(\mathrm{Q})\right] \text {, } \\
& \mathrm{B}_{10}{ }^{\mathrm{loc}}=\mathrm{F}_{\mathrm{I}} \frac{2 \mathrm{il}(\mathrm{I}+\mathrm{1})}{\left(\mathrm{I}+\frac{1}{2}\right)} \frac{\mathrm{y}_{\mathrm{f}}}{\left(\mathrm{x}_{\mathrm{f}}^{2}+\mathrm{y}_{\mathrm{f}}^{2}\right)^{1 / 2}} \mathrm{I}_{1}(\mathrm{Q}),
\end{aligned}
$$

where

$$
Q=2 s\left(1+\frac{1}{2}\left(\frac{x_{f}^{2}+y_{f}^{2}}{w_{0}^{2}}\right)^{1 / 2}\left(1-\frac{2 i s z_{f}}{w_{0}}\right)^{-1}=-i P .\right.
$$

Again E qs. (20) and (21) further simplify when $x_{f} \neq 0, y_{f}$ $=0$ or when $x_{f}=0, y_{f} \neq 0$. Because these special cases are examined at length in Sections 3 and 5, below, we present the simplified expressions here. If $x_{f} \neq 0$ and $y_{f}=0$, Eqs. (20) and (21) become

$$
\begin{aligned}
& A_{I m} \pm l o c=F_{1}\left(\left.\frac{-i}{I+\frac{1}{2}} \frac{X_{f}}{\left|X_{f}\right|}\right|^{m-1}\left[I_{m-1}(Q)+I_{m+1}(Q)\right],\right. \\
& A_{I 0}{ }^{l o c}=F_{1} \frac{2 i l(I+1)}{\left(I+\frac{1}{2}\right)} \frac{x_{f}}{\left|x_{f}\right|} I_{1}(Q), \\
& B_{I m}^{ \pm l o c}=\frac{ \pm F_{1}}{i}\left(\left.\frac{-i}{I+\frac{1}{2}} \frac{x_{f}}{\left|X_{f}\right|}\right|^{m-1}\left[I_{m-1}(Q)-I_{m+1}(Q)\right]\right. \text {, } \\
& \mathrm{B}_{10}{ }^{\mathrm{loc}}=0 \text {, }
\end{aligned}
$$

and if $x_{f}=0$ and $y_{f} \neq 0$, Eqs. (20) and (21) become

$$
\begin{aligned}
A_{I m} \pm l o c & =F_{1}\left|\frac{\mp 1}{I+\frac{1}{2}} \frac{y_{f}}{\left|y_{f}\right|}\right|^{m-1}\left[I_{m-1}(Q)-I_{m+1}(Q)\right], \\
A_{I 0}{ }^{l o c} & =0, \\
B_{I m} \pm l o c & =\frac{ \pm F_{I}}{i}\left(\left.\frac{\mp 1}{I+\frac{1}{2}} \frac{y_{f}}{\left|y_{f}\right|}\right|^{m-1}\left[I_{m-1}(Q)+I_{m+1}(Q)\right],\right. \\
B_{I 0}{ }^{l o c} & =F_{1} \frac{2 i l(I+1)}{\left(I+\frac{1}{2}\right)} \frac{y_{f}}{\left|y_{f}\right|} I_{1}(Q) .
\end{aligned}
$$

In Section 3 we find Eqs. (14), (16), and (18) with Bessel functions of a complex argument to be the most efficient form of the localized model coefficients when the Gaussian beam has spread beyond its focal waist at the position of the particle. Similarly we find Eqs. (20)-(24) with modified Bessel functions of a complex argument to be the most efficient form when the particle is within the focal waist of the beam.

\section{Numerical Implementation of the Localized Model for Gaussian Beam Scattering}

In Section 3 we discuss three numerical aspects of the computation of the far-field scattered intensity of Eqs. (1) and (2). These are (a) the evaluation of $J_{n}(P)$ and $I_{n}(Q)$ in the expressions for the beam-shape coefficients; (b) an algorithm patterned after Wiscombe's method in plane-wave Mie theory ${ }^{3}$ for calculation of the angular functions $\pi_{\mid} \mathrm{m}(\theta)$ and $\tau_{\mid} \mathrm{m}(\theta)$; and (c) the finding that the entire range of $m$ values in Eqs. (2), i.e., $-\mathrm{I} \leq \mathrm{m} \leq \mathrm{I}$, need not be computed. We determine the value of $m_{\max }$ for which inclusion of the terms $-m_{\max } \leq \mathrm{m} \leq$ $m_{\max }$ in Eqs. (2) yields accuracy of 1 in $10^{8}$ in the computation of $\mathrm{I}(\mathrm{r}, \theta, \phi)$.

\section{A. Modified Bessel Functions}

Consider the modified Bessel function $\mathrm{I}_{\mathrm{n}}(\mathrm{Q})$, wheren is a nonnegative integer and $\mathrm{Q}$ is complex. Reference 22 states that for $\operatorname{Re} \mathrm{Q} \leq 12$ or $\operatorname{Re} \mathrm{Q} \leq \mathrm{n}$, the Taylor series expansion

$$
\mathrm{I}_{\mathrm{n}}(\mathrm{Q})=\left(\frac{\mathrm{Q}}{2}\right)^{\mathrm{n}} \sum_{\mathrm{k}=0}^{\infty} \frac{\left(\mathrm{Q}^{2} / 4\right)^{\mathrm{k}}}{\mathrm{k} !(\mathrm{n}+\mathrm{k}) !}
$$

is rapidly convergent, and for $\operatorname{Re} \mathrm{Q}>12$ and $\operatorname{Re} \mathrm{Q}>$ $\mathrm{n}$, the asymptotic series

$$
\begin{aligned}
I_{n}(Q)= & \frac{\exp (Q)}{(2 \pi Q)^{1 / 2}}\left\{1+\sum_{k=1} \frac{(-1)^{k}}{k !(8 Q)^{k}}\left(4 n^{2}-1\right)\right. \\
& \left.\times\left(4 n^{2}-9\right) \ldots\left[4 n^{2}-(2 k-1)^{2}\right]\right\}
\end{aligned}
$$

may be used efficiently. No upper limit for the k sum is given in Eq. (26) because the asymptotic series diverges; i.e., the terms of the series become smaller and smaller, reach a minimum size, and then become larger and larger. Using the remainder theorem for an alternating series, ${ }^{23}$ weachievethe best approximation to $I_{n}(Q)$ when all the terms up to and including one before the smallest term are summed. Examining Eqs. (25) and (26), we found that 1 in $10^{8}$ convergence could be achieved only if Arfken's regions of applicability were changed to $\operatorname{Re} \mathrm{Q} \leq 12$ or $\operatorname{Re} \mathrm{Q} \leq \mathrm{n}$ +2 for Eq. (25) and ReQ $>12$ and $\operatorname{Re} Q>n+2$ for Eq. (26). The upper limit of the k sum in Eq. (25) for 1 in $10^{8}$ convergence was found to be

$$
\mathrm{k}_{\max }=\mathrm{ReQ}+7+0.5|\mathrm{ImQ}| .
$$


For Eq. (26) the upper limit of the k sum for the same convergence criterion was found to be

$$
\mathrm{k}_{\max }=\mathrm{n}+12 \text {. }
$$

F or Im Q $=0$ we checked our computed values of $\mathrm{I}_{\mathrm{n}}(\mathrm{Q}$ by comparing them with the tabulated values in Ref. 24. For Im $\mathrm{Q} \neq 0$, the computed values of $\mathrm{I}_{\mathrm{n}}(\mathrm{Q})$ were checked to make sure that they satisfied the symmetry relation ${ }^{25}$

$$
\mathrm{I}_{\mathrm{n}}\left(\mathrm{Q}^{*}\right)=\mathrm{I}_{\mathrm{n}} *(\mathrm{Q})
$$

The value of $k_{\max }$ in Eqs. (27) and (28) is valid only if $\operatorname{Re} \mathrm{Q} \geq|\mathrm{Im} \mathrm{Q}|$, or when

$$
\left|z_{\mathrm{f}}\right| \leq \frac{\mathrm{w}_{0}}{2 \mathrm{~s}}=\frac{\mathrm{L}}{2},
$$

where $L$ is the spreading length of the Gaussian beam. For $\operatorname{ReQ}<|\mathrm{ImQ}|$ it was found that the number of terms required for convergence rapidly increased, rendering this method of computation inefficient.

Within the beam focal waist given by Eq. (30), if $z_{f}>0$, the beam is converging at the position of the spherical particle and Im Q $>0$. If $z_{f}=0$, the beam focuses in the plane that contains the particle, and Im $\mathrm{Q}=0$. If $\mathrm{z}_{\mathrm{f}}<0$, the beam is diverging at the position of the particle, and Im $\mathrm{Q}<0$. The positioning of the particle in the beam and its corresponding location in the complex Q plane are illustrated in Fig. 2.

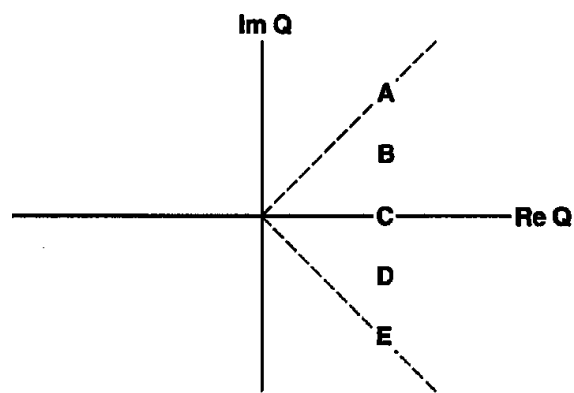

(a)

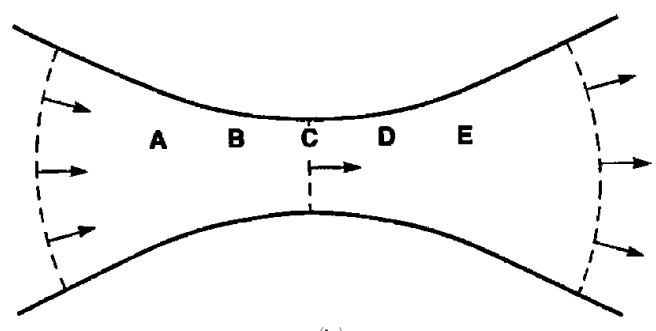

(b)

\section{B. Bessel Functions}

Consider the Bessel function $\mathrm{J}_{\mathrm{n}}(\mathrm{P})$. If $\operatorname{Re} \mathrm{P} \leq 12$ or $\operatorname{Re} \mathrm{P} \leq \mathrm{n}+2$, the Taylor series expansion

$$
J_{n}(P)=\left(\frac{P}{2}\right)^{n} \sum_{k=0}^{\infty} \frac{(-1)^{k}\left(P^{2} / 4\right)^{k}}{k !(n+k) !}
$$

is rapidly convergent. Convergence of 1 in $10^{8}$ was achieved when the upper limit of the $k$ sum was

$$
\mathrm{k}_{\max }=\mathrm{ReP}+12+0.5 \mathrm{Im} \mathrm{P}
$$

for

$$
\left|\mathrm{z}_{\mathrm{f}}\right| \geq \frac{\mathrm{w}_{0}}{2 \mathrm{~s}}=\frac{\mathrm{L}}{2}
$$

or $|\operatorname{Re} P| \geq \operatorname{Im} \mathrm{P} . \quad$ For $\operatorname{Re} \mathrm{P}>12$ and $\operatorname{Re} \mathrm{P}>\mathrm{n}+2$, the asymptotic series is ${ }^{26}$

$$
\begin{aligned}
J_{n}(P)= & \left(\frac{2}{\pi P}\right)^{1 / 2}\left\{\left[1-\frac{\left(4 n^{2}-1\right)\left(4 n^{2}-9\right)}{2 !(8 P)^{2}}\right.\right. \\
& \left.+\frac{\left(4 n^{2}-1\right)\left(4 n^{2}-9\right)\left(4 n^{2}-25\right)\left(4 n^{2}-49\right)}{4 !(8 P)^{4}}-\cdots\right] \\
& \times \cos \left[P-\frac{\left(n+\frac{1}{2}\right) \pi}{2}\right]+\left[0-\frac{\left(4 n^{2}-1\right)}{1 !(8 P)}\right. \\
& \left.+\frac{\left(4 n^{2}-1\right)\left(4 n^{2}-9\right)\left(4 n^{2}-25\right)}{3 !(8 P)^{3}}-\cdots\right] \\
& \left.\times \sin \left[P-\frac{\left(n+\frac{1}{2}\right) \pi}{2}\right]\right)
\end{aligned}
$$

We call the first term in each series in Eq. (34) the $\mathrm{k}=$ -1 term, the second term $\mathrm{k}=1$, the third term $\mathrm{k}=3$, etc. Equation (34) also diverges if too many terms are considered. But when $|\operatorname{Re} P| \geq \operatorname{ImP}$, it converged to 1 in $10^{8}$ for

$$
\mathrm{k}_{\max }=\mathrm{n}+9 .
$$

Again, when $|\operatorname{Re} P|<\operatorname{Im~} \mathrm{P}$, the number of terms in Eqs. (31) and (34) required for 1 in $10^{8}$ convergence grew rapidly, rendering this method of computation inefficient.

For real $P$, we checked our computed values of ${ }_{n}(P)$

Fig. 2. (a) Complex Q plane as defined in Eq. (22). (b) Focused Gaussian beam that is propagating from left to right. The points labeled A through $E$ in the off-axis beam are the positions of the spherical particle within the beam's focal waist. They also correspond to the indicated locations in the complex Q plane. by comparing them with the tabulated values in Ref. 27. If $P$ is complex, care must be taken in the evaluation of Eq. (34). For the trigonometric func- 
tions in Eq. (34), we have

$$
\begin{aligned}
& \cos \left[\mathrm{P}-\frac{\left(\mathrm{n}+\frac{1}{2}\right) \pi}{2}\right] \\
& =\cos \left[\operatorname{Re} P-\frac{\left(\mathrm{n}+\frac{1}{2}\right) \pi}{2}\right] \cosh (\mathrm{ImP}) \\
& -i \sin \left[\operatorname{Re} P-\frac{\left(n+\frac{1}{2}\right) \pi}{2}\right] \sinh (\operatorname{Im} P), \\
& \sin \left[P-\frac{\left(n+\frac{1}{2}\right) \pi}{2}\right] \\
& =\sin \left[\operatorname{Re} P-\frac{\left(n+\frac{1}{2}\right) \pi}{2}\right] \cosh (\operatorname{Im} \mathbf{P}) \\
& +i \cos \left[\operatorname{Re} P-\frac{\left(n+\frac{1}{2}\right) \pi}{2}\right] \sinh (I m P) \text {. }
\end{aligned}
$$

But the $\mathrm{P}^{1 / 2}$ factor in the denominator is potentially problematical because the square root of a complex variable is a two-sheet function. Thus the question arises as to which sheet a particular value of $P$ is on. If the beam focuses upstream from the particle with $\mathrm{Z}_{\mathrm{f}}<0$, Eq. (16) yields $\operatorname{Re} \mathrm{P}>\mathrm{Im} \mathrm{P}$ and I $\mathrm{m} \mathrm{P}>0$. In this region of the complex $\mathrm{P}$ plane adjacent to the positive real axis we have

$$
\begin{aligned}
P & =r e^{i \theta}, \\
P^{1 / 2} & =r^{1 / 2} \exp (i \theta / 2) .
\end{aligned}
$$

On the other hand, if the beam focuses downstream from the particle with $\mathrm{z}_{\mathrm{f}}>0$, Eq. (16) yields $-\operatorname{Re} \mathrm{P}>$ I $\mathrm{m} P$ and I $\mathrm{m} P>0$. The positioning of the particlein the beam and its corresponding location in the complex P planeareillustrated in Fig. 3. Contrary to the situation in Fig. 2, the $z_{f}>0$ region is disjoint from the $\mathrm{Z}_{\mathrm{f}}<0$ region, but satisfies the symmetry relation

$$
(\mathrm{P})_{\mathrm{z}_{\mathrm{f}}>0}=-(\mathrm{P} *)_{\mathrm{z}_{\mathrm{f}}<0}
$$

Thus, rather than computing $J_{n}(P)$ separately for $\mathrm{Z}_{\mathrm{f}}>0$ in the disjoint region, we compute it by the use of the identity 28

$$
\mathrm{J}_{\mathrm{n}}\left[(\mathbf{P})_{\mathrm{z}_{\mathrm{f}}>0}\right]=(-1)^{\mathrm{n}} \mathrm{n}^{*\left[(\mathrm{P})_{\mathrm{z}_{\mathrm{f}}<0}\right]} .
$$

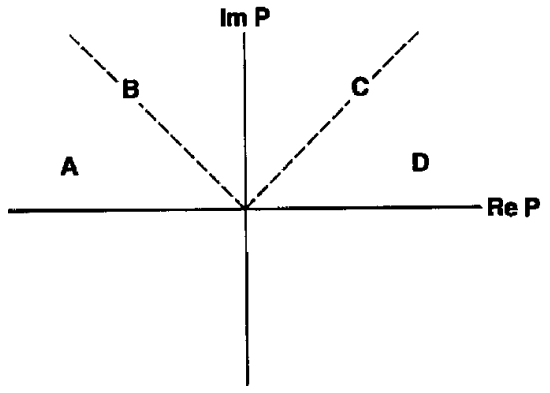

(a)

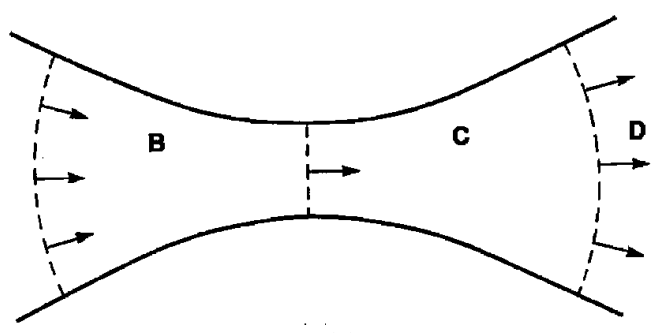

(b)

Fig. 3. (a) Complex P plane as defined in Eq. (16). (b) Focused Gaussian beam that is propagating from left to right. The points labeled A through $D$ in the off-axis beam are the positions of the spherical particle outside the beam's focal waist. A-D also correspond to the indicated locations in the complex $\mathrm{P}$ plane.

\section{Angular Functions}

The angular functions $\pi_{\mid}|\mathrm{m}|(\theta)$ and $\tau_{\mid} \mathrm{m}(\theta)$ satisfy the recursion relations ${ }^{18}$

$$
\begin{aligned}
\pi_{I+1}{ }^{\mathrm{m}}(\theta)= & \frac{2 \mid+1}{1+1-\mathrm{m}} \cos \theta \pi_{\mid}^{\mathrm{m}}(\theta) \\
& -\frac{1+\mathrm{m}}{\mathrm{I}+1-\mathrm{m}} \pi_{\mid-1}^{\mathrm{m}(\theta),} \\
\tau_{l}^{\mathrm{m}}(\theta)= & \mathrm{I} \cos \theta \pi_{\mid}^{\mathrm{m}}(\theta)-(I+\mathrm{m}) \pi_{\mid-1}{ }^{\mathrm{m}}(\theta),
\end{aligned}
$$

with the starting values

$$
\begin{aligned}
\pi_{I-1}{ }^{\prime}(\theta) & =0, \\
\pi_{l}^{\prime}(\theta) & =(2 \mid-1) ! ! \sin ^{1-1} \theta
\end{aligned}
$$

for $m \geq 1$. For $m=0$, the starting values are given in Ref. 18. Often one wishes to calculate the far-field intensity at many angles $\theta$ to construct an angularscattering diagram. Because the computation of $\pi_{1}^{\mathrm{m}}(\theta)$ and $\tau_{1} \mathrm{~m}^{\mathrm{m}}(\theta)$ is within a triple DO LOOP, i.e., $\mathrm{m}, \mathrm{l}$, and $\theta$, savings in computer run time may be realized if the number of multiplications within the triple Do loop is minimized. Following Wiscombe, ${ }^{3}$ for $m \geq 1$ we compute the angular functions with

$$
\begin{aligned}
\mathrm{S} & =\cos \theta \Pi_{l}^{\mathrm{m}}(\theta), \\
\mathrm{T} & =\mathrm{S}-\Pi_{l-1} \mathrm{~m}(\theta), \\
\tau_{l}^{\mathrm{m}}(\theta) & =\frac{\mathrm{I}}{\mathrm{m}} \mathrm{T}-\Pi_{l-1} \mathrm{~m}(\theta),
\end{aligned}
$$


$\Pi_{1+1}{ }^{m}(\theta)=S+\frac{1+m}{I+1-m} T$

where

$$
\Pi_{1}^{\mathrm{m}}(\theta)=m \pi_{1}^{\mathrm{m}}(\theta) .
$$

If the values of $1 / m$ and $I+m /(I+1-m)$ are precalculated, we can use Eqs. (44)-(47) to compute $\Pi_{1+1} \mathrm{~m}(\theta)$ and $\tau_{1} \mid \mathrm{m} /(\theta)$ with 3 multiplications and 3 additions, whereas E qs. (41) and (42) require 6 multiplications and 2 additions. No decrease in the number of multiplications is obtained, however, by computation of the quantities $S_{1}+S_{2}$ and $S_{1}-S_{2}$ rather than computation of $S_{1}$ and $S_{2}$ individually as in Eqs. (49), below.

D. Number of Terms in the $m$ Sum

In previous Gaussian beam-scattering cal culations, ${ }^{18,29}$ it has been useful to interchange the order of thel and m sums in Eqs. (2), yielding

$$
\begin{aligned}
& \mathrm{S}_{1}(\theta, \phi)=\sum_{\mathrm{I}=1}^{I_{\max }} \frac{2 \mathrm{l}+1}{2 \mathrm{l}(\mathrm{I}+1)} \mathrm{B}_{\mid \mathrm{I}} \mathrm{b}_{\mathrm{I}} \tau_{\mathrm{I}} \mathrm{O}^{0}(\theta) \\
& +\sum_{m=1}^{m_{\max }} \sum_{\mathrm{l}=\mathrm{max}}^{I_{\max }} \frac{2 \mathrm{l}+1}{2 \mathrm{l}(\mathrm{l}+1)} \mathrm{i} \mathrm{a}_{\mathrm{l}} \Pi_{\mathrm{l}}{ }^{\mathrm{m}}(\theta) \\
& \times\left[-A_{I m}{ }^{+} \exp (i m \phi)+A_{l m}{ }^{-} \exp (-i m \phi)\right] \\
& +\sum_{\mathrm{m}=1}^{\mathrm{m}_{\max }} \sum_{\mathrm{l}=\mathrm{m}}^{I_{\max }} \frac{2 \mathrm{l}+1}{2 \mathrm{l}(\mathrm{I}+1)} \mathrm{b}_{\mathbf{l}} \tau_{l}{ }_{l}^{\mathrm{m}}(\theta) \\
& \times\left[B_{I m}{ }^{+} \exp (i m \phi)+B_{I m}{ }^{-} \exp (-i m \phi)\right] \\
& \mathrm{S}_{2}(\theta, \phi)=\sum_{\mathrm{l}=1}^{I_{\max }} \frac{2 \mathrm{l}+1}{2 \mathrm{l}(\mathrm{I}+1)} \mathrm{A}_{10} \mathrm{a}_{1} \tau_{\mathrm{I}}{ }^{0}(\theta) \\
& +\sum_{m=1}^{m_{\max }} \sum_{\mathrm{I}=\mathrm{m}}^{I_{\max }} \frac{2 \mathrm{l}+1}{2 \mathrm{l}(\mathrm{I}+\mathrm{l})} \mathrm{a}_{\mathrm{l}} \tau_{\mathrm{l}} \mathrm{m}(\theta) \\
& \times\left[A_{I m}{ }^{+} \exp (i m \phi)+A_{I m}{ }^{-} \exp (-i m \phi)\right] \\
& +\sum_{m=1}^{m_{\max }} \sum_{\mathrm{l}=\mathrm{m}}^{I_{\max }} \frac{2 \mathrm{l}+1}{2 \mathrm{l}(\mathrm{I}+1)} \mathrm{i} \mathrm{b}_{\mathbf{l}} \Pi_{l}{ }^{\mathrm{m}}(\theta) \\
& \times\left[B_{I m}{ }^{+} \exp (i m \phi)-B_{I m}{ }^{-} \exp (-i m \phi)\right] \text {, }
\end{aligned}
$$

where the largest partial wave $I_{\max }$ is given by 3,4

$$
I_{\max }=2+X+4.3 X^{1 / 3},
$$

the size parameter $X$ is

$$
X=\frac{2 \pi a}{\lambda},
$$

and the value of $m_{\max }$ is yet to be determined. For the examination of Iow-order MDR's, the value of $I_{\max }$ may have to be increased somewhat. ${ }^{3}$ Numerically it has been found that as $m$ increases for a fixed value of $\mathrm{I}, \mathrm{A}_{\mathrm{Im}^{ \pm}}$and $\mathrm{B}_{\mathrm{Im}^{ \pm}}{ }^{ \pm}$rapidly decrease, and $\Pi_{\mid} \mathrm{m}(\theta)$ and $\tau_{1} \mathrm{~m}(\theta)$ rapidly increase, but the product of the beamshape coefficients and the angular functions, which we call the weighted beam-shape coefficients, also rapidly decreases. ${ }^{18}$ This permits truncation of the $m$ sums in Eqs. (49) at $m_{\max } \ll I$ with little loss in accuracy.

This result may be demonstrated analytically as follows. Consider for simplicity the case $z_{f}=0$ so that $\mathrm{Q}$ is real. Consider also a relatively high partial wave, a small beam focal waist, or a relatively large off-center impact of the beam on the sphere so that $\mathrm{Q}>$ 12. If $\mathrm{x}_{\mathrm{f}} \neq 0$ and $\mathrm{y}_{\mathrm{f}}=0$, the beam-shape coefficients are given by Eqs. (23). We now examine these expressions as a function of $m$. When $m$ is small, $I_{m \pm 1}(Q)$ may be approximated by the first two terms of Eq. (26), yielding

$$
\begin{aligned}
\left|A_{I \mathrm{~m}} \pm \mathrm{loc}\right| \approx & \frac{\exp \left[-\mathrm{s}^{2}\left(I+\frac{1}{2}\right)^{2}\right] \exp \left(-\mathrm{x}_{\mathrm{f}}^{2} / \mathrm{w}_{0}^{2}\right)}{\left(1+\frac{1}{2}\right)^{\mathrm{m}-1}} \\
& \times(2 \pi \mathrm{Q})^{-1 / 2} \exp (\mathrm{Q})\left(2-\frac{3}{4 Q}-\frac{\mathrm{m}^{2}}{\mathrm{Q}}\right), \\
\left|\mathrm{B}_{\mid \mathrm{m}} \pm\right| \mathrm{loc} \mid \approx & \frac{\exp \left[-\mathrm{s}^{2}\left(I+\frac{1}{2}\right)^{2}\right] \exp \left(-\mathrm{x}_{\mathrm{f}}^{2} / \mathrm{w}_{0}^{2}\right)}{\left(1+\frac{1}{2}\right)^{\mathrm{m}-1}} \\
& \times(2 \pi \mathrm{Q})^{-1 / 2} \exp (\mathrm{Q})\left(\frac{2 \mathrm{~m}}{\mathrm{Q}}\right) .
\end{aligned}
$$

Similar expressions occur if $x_{f}=0$ and $y_{f} \neq 0$. For $\theta$ away from $0^{\circ}$ or $180^{\circ}$ and for large I, the angular functions approach the asymptotic values ${ }^{18}$

$$
\begin{aligned}
\Pi_{l} \mathrm{~m}(\theta) \rightarrow & \left(\frac{\mathrm{m}}{\mathrm{I}}\right)\left(\frac{2}{\pi}\right)^{1 / 2} I^{\mathrm{m}+1 / 2}(\sin \theta)^{-3 / 2} \\
& \times \cos \left[\left(I+\frac{1}{2}\right) \theta+\frac{\mathrm{m} \pi}{2}-\frac{\pi}{4}\right], \\
\tau_{I}^{\mathrm{m}}(\theta) \rightarrow & \left(\frac{2}{\pi}\right)^{1 / 2} I^{\mathrm{m}+1 / 2}(\sin \theta)^{-1 / 2} \\
& \times \sin \left[\left(I+\frac{1}{2}\right) \theta+\frac{\mathrm{m} \pi}{2}-\frac{\pi}{4}\right]
\end{aligned}
$$

or, retaining only the powers of I in expressions (53),

$$
\left|\tau_{l}^{\mathrm{m}}(\theta)\right| \approx \frac{\mathrm{I}}{\mathrm{m}}\left|\Pi_{l}^{\mathrm{m}}(\theta)\right| \approx \mathrm{I}^{\mathrm{m}+1 / 2} .
$$

We can now see from expressions (52) that the beam-shape coefficients decrease as a function of $m$ as $\mathrm{I}^{-\mathrm{m}+1}$, whereas from expression (54) the angular functions increase as $I^{m+1 / 2}$. Thus for small $m$ the dominant $\mathrm{m}$ dependence of the two contributions cancels, and the weighted beam-shape coefficient 
$\left|A_{I m}{ }^{ \pm l^{\prime} c_{\tau_{l}} m}\right|$ of Eqs. (49) decreases only quadratically in m. Likewise $\left|B_{\mid m^{ \pm}}{ }^{ \pm 0} c_{\tau_{1}} m\right|$ starts out much smaller and increases linearly in $m$. Because from expression (54), $m \pi_{\mid m} \ll \tau_{l}^{m}$ for small $m$, the weighted beam-shape coefficients $\left|A_{\mid m^{m}}{ }^{ \pm 0 c} m \pi_{\mid} m\right|$ and $\left|B_{\mid m^{m}}{ }^{ \pm 0 c} m \pi_{\mid} m\right|$ are much smaller than $\mid A_{\mid \mathrm{m}}{ }^{ \pm \mathrm{Ioc}_{\tau_{1}} \mathrm{~m} \mid}$ and $\mid \mathrm{B}_{\mid \mathrm{m}}{ }^{ \pm \mathrm{loc}_{\tau_{1}} \mathrm{~m} \mid}$.

When $m$ is large, $I_{m \pm 1}(Q)$ may be approximated with Eq. (25) as

$$
\begin{aligned}
\left|A_{I \mathrm{~m}} \pm \mathrm{loc}\right| \approx\left|\mathrm{B}_{\mathrm{Im}} \pm \mathrm{loc}\right| & \approx \frac{\exp \left[-\mathrm{s}^{2}\left(I+\frac{1}{2}\right)^{2}\right] \exp \left(-\mathrm{x}_{\mathrm{f}}^{2} / \mathrm{w}_{0}^{2}\right)}{\left(I+\frac{1}{2}\right)^{\mathrm{m}-1}} \\
& \times \frac{(\mathrm{Q} / 2)^{\mathrm{m}-1}}{(\mathrm{~m}-1) !} \exp \left(\mathrm{Q}^{2} / 4 \mathrm{~m}\right) .
\end{aligned}
$$

A similar expression occurs if $x_{f}=0$ and $y_{f} \neq 0$. Thus the weighted beam-shape coefficients are approximately equal and rapidly decrease when $\mathrm{Q}^{2} \ll 4 \mathrm{~m}$. To check this predicted dependence of the weighted beam-shape coefficients on $\mathrm{m}$, we computed $\left|A_{\mid \mathrm{m}}{ }^{ \pm \operatorname{loc}_{\tau_{1}} \mathrm{~m}}\right|$ and $\mid \mathrm{B}_{\mid \mathrm{m}}{ }^{ \pm \mathrm{loc}_{\tau \mid} \mathrm{m} \mid}$ numerically with Eqs. (23), (25)-(28), and (48) for $\mathrm{I}=430, \mathrm{x}_{\mathrm{f}}=42.5 \mu \mathrm{m}, \mathrm{y}_{\mathrm{f}}=\mathrm{z}_{\mathrm{f}}=0, \mathrm{w}_{0}=$ $13.3 \mu \mathrm{m}$, and $\lambda=0.6328 \mu \mathrm{m}$ corresponding to $\mathrm{Q}=$ 20.83. The results are shown in Fig. 4. As predicted in expressions (52), initially $\left|\mathrm{A}_{\mid \mathrm{m}}{ }^{ \pm \mathrm{loc}_{\tau} \mathrm{m}} \mathrm{m}\right|$ is large and slowly decreasing, and $\left|\mathrm{B}_{\mid \mathrm{m}}{ }^{ \pm \mathrm{loc}_{\tau} \mathrm{m}}\right|$ is small and increasing. For $\mathrm{m} \gtrsim 14$ the two weighted beam-

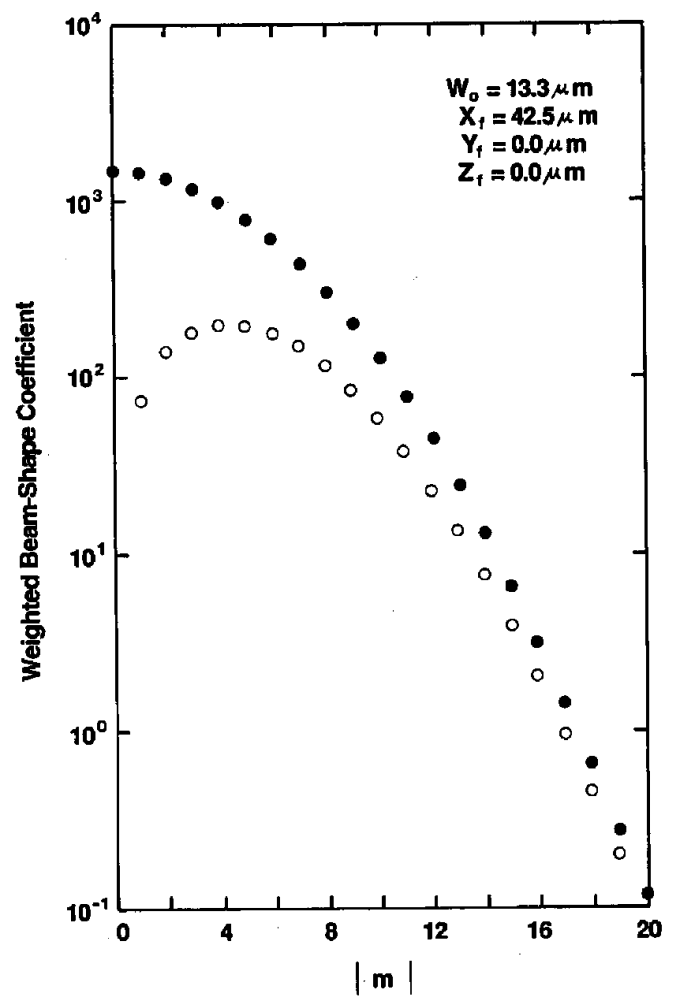

Fig. 4. Weighted beam-shape coefficients $\left|A_{l m}{ }^{l o c_{\tau}} m\right|$ (filled circles) and $\left|B_{\mid m}{ }^{l o c} \tau\right| m \mid$ (open circles) as a function of $|\mathrm{m}|$ for $\mathrm{I}=430$ and an off-axis Gaussian beam with $\lambda=0.6328$. shape coefficients are roughly equal and decrease almost exponentially as a function of $m$.

Figure 4 suggests that the $m$ sum in Eqs. (49) may be cut off for large $Q$ and $I$ at $m_{\max } \ll I$ with minimal loss in accuracy. The cutoff value $m_{\max }$ was determined by the criterion

$$
\begin{aligned}
\frac{\left|A_{I m}{ }^{ \pm l o c_{\tau_{l}} m}\right|_{m_{\max }}}{\left|A_{I m} \pm l o c_{\tau_{l}} m\right|_{\text {small } m}}= & \frac{2\left(\frac{Q}{2}\right)^{m_{\max }-1}}{\left(m_{\max }-1\right) !} \exp \left(Q^{2} / 4 m_{\max }\right) \\
& \times(2 \pi Q)^{1 / 2} \exp (-Q) \leqslant 10^{-8} .
\end{aligned}
$$

The solution of Eq. (56) was obtained numerically, and it is well approximated by the relation

$$
\mathrm{m}_{\max }=6.5 \mathrm{Q}^{1 / 2} \quad \text { for } 6 \leq \mathrm{Q} \leq 40 .
$$

This result was tested with Eqs. (20)-(22) and (25)-(28) and expression (54) to compute the weighted beamshape coefficients. Equation (57) was found to be accurate in every instance. When $\mathrm{Q}$ was made complex, the weighted beam-shape coefficients fell by a factor of $10^{8}$ when

$$
\begin{aligned}
\mathrm{m}_{\max }=\left(6.5+2.0 \frac{\mid \mathrm{Im} \mathrm{Q}}{\operatorname{ReQ}}\right) & (\operatorname{Re~Q})^{1 / 2} \\
& \text { for }|\mathrm{Im} \mathrm{Q}| \leq \operatorname{ReQ} .
\end{aligned}
$$

As a final check of Eqs. (25)-(28), (31) and (32), (34)-(40), and (58) we calculated the far-field scattered intensity for $z_{f}=w_{0} / 2 s$, the boundary between the modified Bessel function representation and the Bessel function representation of the beam-shape coefficients. The far-field scattered intensity was calculated with each representation, and the results agreed to better than 1 in $10^{8}$.

\section{E. Inclusion of the Incident Beam}

Before we examine the run time of our Gaussian beam-scattering computer program, an important addition to the scattering amplitudes of Eqs. (2) and (49) must be made. Equations (2) and (49) give us the amplitude for the far-field scattered portion of the electromagneticfiel ds exterior to the spherical particle. But the entire exterior field, the scattered field plus the incident field, is measured in the experiments. Thus the amplitude of the incident field should be appended to Eqs. (2) to have the resultant expression agree with experimental observations. The incident beam is included in far-field plane-wave Mie theory only at $\theta=0$ but is in included in the near-forward direction in the near field. 30 For Gaussian beam scattering the incident beam must be included in both the near field and the far field in the near-forward direction because of the spreading of the incident beam. ${ }^{31,32}$

In particular consider a narrow Gaussian beam that is incident only slightly off-axis upon a large particle. Because the particle obstructs most of the incident beam, thenear-forward diffracted fiel d should 
be quite weak because only the tail of the Gaussian beam passes the edge of the particle. ${ }^{31}$ Yet the numerical implementation of Eqs. (1), (2), and (49) yields a large unobserved diffraction like peak in the near-forward direction, ${ }^{32}$ which is canceled by the spreading of the incident beam. The addition of the incident beam to the scattering amplitudes for an off-axis beam is given by

$$
\begin{aligned}
& S_{1}{ }^{\text {total }}(\theta, \phi)= \begin{cases}S_{1}{ }^{\text {scattered }}(\theta, \phi) & \text { for } \theta>10 \mathrm{~s} \\
S_{1}{ }^{\text {scattered }}(\theta, \phi) & \\
-\sin \phi S^{\text {incident }}(\theta, \phi) & \text { for } \theta \leq 10 \mathrm{~s},\end{cases} \\
& S_{2}{ }^{\text {total }}(\theta, \phi)= \begin{cases}S_{2}{ }^{\text {scattered }}(\theta, \phi) & \text { for } \theta>10 \mathrm{~s} \\
S_{2}{ }^{\text {scattered }}(\theta, \phi) & \\
-\cos \phi S^{\text {incident }}(\theta, \phi) & \text { for } \theta \leq 10 \mathrm{~s},\end{cases} \\
& \operatorname{Sincident}(\theta, \phi)=\frac{1}{2 \mathrm{~s}^{2}} \exp \left(-\theta^{2} / 4 \mathrm{~s}^{2}\right) \\
& \times \exp \left[-\frac{i \theta}{s}\left(\frac{x_{f}}{w_{0}} \cos \phi+\frac{y_{f}}{w_{0}} \sin \phi\right)\right] \\
& \times \exp \left(-i z_{f} / s w_{0}\right) \exp \left(i \theta^{2} z_{f} / 2 s w_{0}\right) \text {. }
\end{aligned}
$$

We obtain the corresponding equations for an on-axis beam from Eqs. (59) by setting $x_{f}=y_{f}=0$. The inclusion of the incident beam is implemented in our Gaussian beam-scattering computer program.

\section{Timing Study of the Localized Model of Gaussian Beam Scattering}

To assess the performance of our Gaussian beamscattering program, we tested it on the situation in which $\mathrm{a}=50 \mu \mathrm{m}, \mathrm{n}=1.333, \lambda=0.6328 \mu \mathrm{m}, \mathrm{w}_{0}=10$ $\mu \mathrm{m}, \mathrm{x}_{\mathrm{f}}=\mathrm{z}_{\mathrm{f}}=0, \mathrm{y}_{\mathrm{f}}=20 \mu \mathrm{m}, \phi=90^{\circ}$ and for 361 values of $\theta$ in the interval $-180^{\circ} \leq \theta \leq 180^{\circ}$. The size parameter for this case is $X=496.46$, the largest partial wave is $I_{\max }=532$, the degree of beam confinement is $S=0.01$, and the off-centeredness of the beam is $\mathrm{Q}_{\max }=21.3$. According to Eq. (57) this value of $\mathrm{Q}_{\max }$ corresponds to $\mathrm{m}_{\max }=30$, which was used as the upper limit of the $m$ sum in Eqs. (49). This program, as well as all the other programs for which timing studies were made, was run on a Compaq 386-33 MHz personal computer equipped with a Weitek numerical coprocessor. The run time of the localized model Gaussian beam program was $195 \mathrm{~s}$ for the parameters given above. Less than $1 \mathrm{~s}$ of this time was spent computing the incident beam of Eqs. (59) and the Mie partial-wave scattering amplitudes for $1 \leq \mathrm{I} \leq \mathrm{I}_{\max }$. Because the localized approximation replaces the numerical integrations of Eqs. (5), only $9 \mathrm{~s}$ were spent computing $A_{\mid m^{ \pm}}{ }^{ \pm 10 c}$ and $B_{\mid m}{ }^{ \pm 10 c}$ for 1 $\leq \mathrm{I} \leq \mathrm{I}_{\max }$ and $0 \leq \mathrm{m} \leq \mathrm{m}_{\max }$ with Eqs. (24). The program spent 3:05, or almost $95 \%$ of the run time, computing $\pi_{l} \mathrm{~m}(\theta)$ and $\tau_{l} \mathrm{~m}(\theta)$, multiplying the beamshape coefficients by the angular functions, and adding everything together to obtain the scattering amplitudes. This division of run time is similar to that reported by Wiscombe for plane-wave Mietheory. ${ }^{3}$ When $z_{f} \neq 0$, the beam-shape coefficients were calculated with Eqs. (20) and (21) rather than the simpler Eqs. (24). The fact that $Q$ was complex meant that 20 $s$ more was required for the calculation of $A_{1 \mathrm{~m}}{ }^{ \pm \mathrm{loc}}$ and $B_{I m} \pm$ loc for $1 \leq 1 \leq I_{\max }$ and $0 \leq m \leq m_{\max }$. The time for all the other computations was unchanged. For comparative purposes, a plane-wave Mietheory calculation for $\mathrm{a}=50 \mu \mathrm{m}, \mathrm{n}=1.333, \lambda=0.6328 \mu \mathrm{m}$ and for 361 values of $\theta$ in the interval $-180^{\circ} \leq \theta \leq 180^{\circ}$ took slightly less than $3 \mathrm{~s}$ on the same computer. Thus our Gaussian beam program runs almost 70 times slower than Mie theory for these parameters.

In Eqs. (2), the full range of $I$ and $m$ values is $1 \leq$ $\mathrm{I} \leq \mathrm{I}_{\max }$ and $-\mathrm{I} \leq \mathrm{m} \leq \mathrm{I}$. For the parameters of our numerical experiment this requires the computation and storage of 568,178 beam-shape coefficients. Truncating the $\mathrm{m}$ sum at $\mathrm{m}_{\max }=30$ reduces the number to 63,166 , which is $11.1 \%$ of the total. Using the symmetry-relations for $A_{1 m}{ }^{+l o c}$ and $A_{1 m}{ }^{-10 c}$ and for $B_{I m}{ }^{+l o c}$ and $B_{I m}{ }^{-10 c}$ of $E$ qs. (14), (18), (20), and (21) further reduces the number of coefficients computed to 32,116 , which is $5.6 \%$ of the total. Thus the truncation of the $\mathrm{m}$ sum at 1 in $10^{8}$ accuracy for the far-field intensity represents a substantial savings in computer run time in this example.

A program that also computes the scattering amplitudes with Eqs. (49) but computes $A_{I m}$ and $B_{I m}$ with numerical integration of Eqs. (5) was written. The grid size for the $\theta$ and $\phi$ integrations required for convergence of the numerical integrations is given elsewhere. ${ }^{18}$ For the parameters of our numerical experiment with $m_{\max }=30$, the run time for this program when 32,116 beam-shape coefficients were used was $4.5 \mathrm{~h}$ which is a factor of 83 slower than our localized model program. If the full range of $m$ values had been used, the run time would have been longer by another factor of at least 17.8.

In Refs. 9-11 the incident Gaussian beam is expanded in an angular spectrum of plane waves. The plane waves are then decomposed into vector spherical harmonics. We obtain the total vector spherical harmonic coefficients by summing the individual plane-wave coefficients over the angular spectrum. The total vector spherical harmonic coefficients are then input into a T-matrix program for calculation of either the far-field intensity or the interior source function. ${ }^{29}$ The computer run time required for the computation of each set of total vector spherical harmonic coefficients $a_{e m n}{ }^{t}, a_{o m n}{ }^{t}, b_{e m n}{ }^{t}$, and $b_{o m n}{ }^{t}$ was $1.57 \mathrm{~s}$. Thus computation of the 32,116 sets of coefficients required for our test situation takes $14 \mathrm{~h}$.

The Rouen computer program for Gaussian beam scattering described in Ref. 19 is in many aspects similar to the program that we have described here. The localized approximation is used and Eqs. (7) are written as a single sum over $\mathrm{j}$. But (a) the sum was not recognized as being a Bessel function or a modified Bessel function, (b) the series was truncated when an individual term fell below $10^{-30}$ rather than at 1 in $10^{8}$ accuracy, and (c) the number of $m$ values 
was set at $m_{\max }=10$ rather than at $m_{\max }$ of Eqs. (57)-(58). This resulted in $A_{1 m}{ }^{10 c}$ and $B_{1 m}$ loc being computed to much greater than 1 in $10^{8}$ accuracy. But for $\mathrm{Q}>2.4$ a number of weighted beam-shape coefficients were omitted that were larger than $10^{-8}$ of the coefficients that were included. For the parameters of our numerical experiment, the Rouen program computed the localized approximation beamshape coefficients in $96 \mathrm{~s}$ which is a factor of 10.7 slower than with our program. But, when the localization approximation is used, the most time-consuming part of the program is the computation of $\tau_{1} \mathrm{~m}$, and $\pi_{l}{ }^{m}$. Thus the entire Rouen program is only a factor of 1.45 slower than ours.

The run-time study described here was for only one particular example of Gaussian beam scattering, and it would be unwarranted to extrapolate the comparison between the various computational schemes to all cases of Gaussian beam scattering without further testing. For example, consider the published calculations of Gaussian beam scattering given in Table 1. Of particular interest in Table 1 are the values of $I_{\max }$, the highest partial wave in the computation, and $\mathrm{Q}_{\max }$, which is a measure of the highest partial wave, the extent of beam focusing, and the degree of offcenteredness of the incident beam. In each of the references cited in Table 1, the authors had a different goal in mind when performing the calculations, thus dictating different choices for $I_{\max }$ and $Q_{\max }$. L Lock ${ }^{18}$ was interested in rainbow formation and thus re quired a large particle, i.e., $I_{\max }=565$. Because the particle was large, the incident beam did not need to be tightly focused, i.e., $Q_{\max }=9$, to see the effect for which he was looking. The combination of large $I_{\max }$ and relatively small $Q_{\max }$ is tailor-made for both the localized model and the truncation of the $m$ sum because computing the full range of $A_{I m}$ and $B_{I m}$ coefficients by numerical integration for large I would take a prohi bitively long time on a personal computer. The same consideration holds true in computation of calibration curves for particle-sizing instruments in the large-particle regime. ${ }^{36}$

Barton et al.8,33,34 were interested in smaller particles, i.e., $I_{\max } \approx 45$. But for them to see the effects that they were looking for, the incident beam had to be more tightly focused, i.e., $Q_{\max } \approx 37$. Their beam localization of $s=0.084$ is near the limit of the validity of the localized model for an off-axis beam, so

Table 1. Parameters in Published Gaussian Beam-Scattering Calculations

\begin{tabular}{cclccrr}
\hline $\begin{array}{c}\text { Reference } \\
18\end{array}$ & $\mathrm{a}(\mu \mathrm{m})$ & $\lambda(\mu \mathrm{m})$ & $\mathrm{w}_{0}(\mu \mathrm{m})$ & $\mathrm{s}$ & $\mathrm{I}_{\max }$ & $\mathrm{Q}_{\max }$ \\
\hline 8 & 43.3 & 0.5145 & 20.0 & 0.004 & 565 & 9.0 \\
\hline 33 & 2.5 & 1.06 & 2.0 & 0.084 & 27 & 5.8 \\
34 & 2.5 & 1.06 & 2.0 & 0.084 & 44 & 37.4 \\
10 & 8.0 & 1.06 & 2.0 & 0.084 & 64 & 86.5 \\
11 & 8.0 & 1.06 & 2.0 & 0.084 & 64 & 64.9 \\
& 13.4 & & & & 100 & 113.4 \\
35 & 9.45 & 1.06 & 2.0 & 0.084 & 74 & 88.7 \\
19 & 38.08 & 0.5145 & 10.0 & 0.0082 & 500 & 8.2 \\
\hline
\end{tabular}

its use still yields a great decrease in computer run time. But now because $m_{\max } \approx 40$, there is not much point in truncating the $m$ sum before $m=1$. Barton et al. performed their computations on a Silicon Graphics 4D/380 VGX Super Computer, ${ }^{37}$ taking advantage of the much faster speed of 34 MFlops to perform the numerical integrations for $A_{I m}$ and $B_{I m}$ in Eqs. (5). Khaled et al. ${ }^{10,11,35}$ were also interested in small particles, i.e., $I_{\max } \approx 75$, and in particular with MDR's excited by a tightly focused beam, $s=0.084$, that was incident upon a spherical particle somewhat beyond the particle's edge with $\mathrm{Q}_{\max } \approx 100$. Again the $s$ value is just within the range of applicability of the localized model, but truncation of the $m$ sum again is not possible. If the beam had been even more tightly focused, with $\mathrm{s}>0.1$, the localized beam model might produce a relatively poor approximation to the actual beam profile. ${ }^{15}$

The moral of the story is that the computational cost of Gaussian beam-scattering calculations depends on the size of the spherical particle through $I_{\max }$, on the degree of focusing and the degree of off-centeredness of the incident beam through $\mathrm{Q}_{\max }$, and on the speed of one's computer. The computational method described here provides the greatest computational savings for $\mathrm{Q}_{\max } \lesssim 40$ and large $I_{\max }$, thereby permitting the computation to be easily handled by a personal computer. For much larger values of $Q_{\max }$ or smaller values of $I_{\max }$, the computational savings may not be as great. But the calculation is still very efficient on a personal computer.

\section{MDR Excitation in the Localized Model}

It has been found both experimentally ${ }^{38-40}$ and theoretically ${ }^{10,33}$ within the last few years that one can greatly enhance the MDR excitation rate in a dielectric microparticle by having a tightly focused Gaussian beam that is incident somewhat beyond the microparticle's edge. Theoretically the reason for this is that the spherical Bessel function $j_{1}(n k r)$ inside the particle couples to the spherical Bessel function $\mathrm{j}_{\text {I }}(\mathrm{kr})$ outside the particle. ${ }^{10}$ Because the energy density of a MDR is largest just inside the particle surface, we must have $I \geqslant n k a$ to make $j_{l}(n k r)$ reach its peak value there. ${ }^{41}$ But because the classical impact parameter $b$ of the geometric light ray associated with the particle wavel is $42,43 \mid \approx k b$, we needed $\mathrm{b} / \mathrm{a} \leqslant \mathrm{n}$ for the excitation of the MDR. This impact parameter describes a light ray that passes the particle somewhat beyond its edge.

Thelocalized model permits a more detailed description of the effect. Consider a focused Gaussian beam with $\mathrm{x}_{\mathrm{f}} \neq 0$ and $\mathrm{y}_{\mathrm{f}}=\mathrm{z}_{\mathrm{f}}=0$. Assume that the particle's radius and refractive index and the beam's wavelength are such that the Mie particle-wave scattering amplitude $a_{1}$ resonates, producing a TM-type MDR. In Eqs. (4) the effect that $a_{1}$ has on the far-field intensity is modulated by the beam-shape coefficients $A_{I m}$ for $-I \leq m \leq I$. If the $A_{I m}$ are small, the MDR is suppressed. If the $A_{I m}$ are large, the 
strength of the MDR is enhanced. The same holds true for the interior source function because the Mie interior amplitude $c_{1}$ and the scattering amplitude $a_{1}$ resonate simultaneously and because the interior field is proportional to $c_{1} A_{I m}$. As can be seen in $E$ qs. (23) and Fig. 4, when $x_{f} \neq 0$ and $y_{f}=0$, we have $A_{I m}{ }^{l o c} \gg B_{I m}$ loc for small $m$. Thus the TM resonances are orders of magnitude stronger than the TE resonances. On the other hand, when $x_{f}=0$ and $y_{f} \neq 0$, Eqs. (24) show that $B_{\mid m}{ }^{l o c} \gg A_{I m}{ }^{\text {loc }}$ for small $m$. Thus the TE resonances that are proportional to $B_{I m}{ }^{10 c} b_{1}$ are orders of magnitude stronger than the TM resonances.

Consider the weighted beam-shape coefficient $A_{I m}{ }^{ \pm l o c_{T}}{ }^{m}$ of Eqs. (23) and expression (54). When $m$ is small and $I_{m \pm 1}(Q)$ may be approximated by the first two terms of Eq. (26), we have

$$
\begin{aligned}
\left|A_{I m} \pm l o c_{\tau_{l}} m\right| \approx & 1\left(4 \pi s \mid \frac{x_{f}}{w_{0}}\right)^{-1 / 2} \exp \left\{-\left[s\left(I+\frac{1}{2}\right)-\left|\frac{x_{f}}{w_{0}}\right|^{2}\right\}\right. \\
& \times\left(2-\frac{3}{4 Q}-\frac{m^{2}}{Q}\right) .
\end{aligned}
$$

As seen in Fig. 4, Eq. (60) decreases quadratically as a function of $\mathrm{m}$. But as a function of $\mathrm{x}_{\mathrm{f}} \mathrm{Eq}$. (60) peaks at

$$
I+\frac{1}{2} \approx \frac{\left|x_{f}\right|}{w_{0} S}=\frac{2 \pi}{\lambda}\left|x_{f}\right| .
$$

Because the resonating partial wave I for a low MDR order number i occurs for ${ }^{44,45}$

$$
\begin{aligned}
& \mathrm{nx}=\left(I+\frac{1}{2}\right)+\alpha_{i}\left(I+\frac{1}{2}\right)^{1 / 3} 2^{-1 / 3}-\mathrm{V}\left(\mathrm{n}^{2}-1\right)^{-1 / 2}, \\
& \mathrm{~V}= \begin{cases}\mathrm{n} & \text { for a TE resonance } \\
\frac{1}{\mathrm{n}} & \text { for a TM resonance }\end{cases}
\end{aligned}
$$

where ${ }^{46} \mathrm{Ai}\left(-\alpha_{\mathrm{i}}\right)=0$, the greatest enhancement in the strength of the MDR occurs for an incident beam with

$$
\begin{aligned}
& \frac{y_{f}}{a} \approx n-\alpha_{i}\left(\frac{n}{2}\right)^{1 / 3} \frac{1}{x^{2 / 3}}+\frac{n}{\left(n^{2}-1\right)^{1 / 2} x}+\frac{1}{6}\left(\frac{2}{n}\right)^{1 / 3} \frac{\alpha_{i}^{2}}{x^{4 / 3}} \\
& \text { for a TE resonance } \\
& \frac{x_{f}}{a} \approx n-\alpha_{i}\left(\frac{n}{2}\right)^{1 / 3} \frac{1}{x^{2 / 3}}+\frac{1}{n\left(n^{2}-1\right)^{1 / 2} x}+\frac{1}{6}\left(\frac{2}{n}\right)^{1 / 3} \frac{\alpha_{i}^{2}}{x^{4 / 3}} \\
& \text { for a TM resonance, }
\end{aligned}
$$

or for focusing somewhat outside the edge of the partide. As an example, the $\mathrm{TE}_{58,1}$ resonance has $\mathrm{I}=58, \mathrm{i}=1$, and $\mathrm{X}=47.3094299$ for $\mathrm{n}=1.36$. Expressions $(63)$ yield $\mathrm{y}_{\mathrm{f}} / \mathrm{a}=1.24$, which agrees well with the value of 1.23 in figure $2 \mathrm{~b}$ of Ref. 10 . Similarly the $\mathrm{TM}_{34,1}$ resonance has $\mathrm{I}=34, \mathrm{i}=1$, and $\mathrm{X}=29.753$ for $\mathrm{m}=1.33$. Expressions (63) yield $\mathrm{x}_{\mathrm{f}} / \mathrm{a}=1.16$, which agrees well with thevalue of approximately 1.14 in figure 7 of Ref. 33. Also the $T E_{34,1}$ resonance has $I=34, i=1$, and $X=29.365$ for $m=1.33$. Expressions (63) yield $\mathrm{y}_{\mathrm{f}} / \mathrm{a}=1.18$, which agrees well with the value of approximately 1.18 in figure 8 of Ref. 33. Expression (60) also shows that the low azimuthal modes $m$ of the MDR are all excited to nearly the same extent, whereas the degree of excitation of the high azimuthal modes fall off rapidly. This is illustrated in Fig. 5.

In summary, the most important result of this paper is that Gouesbet's localized model for the beam-shape coefficients in scattering of a focused Gaussian beam by a spherical particlemay be written in terms of either Bessel functions or modified Bessel functions. On the one hand this simplified form leads to the construction of a fast-running computer program for Gaussian beam scattering that can be implemented on a personal computer. On the other hand the simplified form provides a simple analytical formula for the beam-shape coefficients that permits one to obtain an intuition of various effects that occur in Gaussian beam scattering.

This work was supported in part by National Aeronautics and Space Administration grant NCC3204. I thank Scott Schaub of Schuller International, Mountain Technical Center, and formerly of the University of Nebraska-Lincoln, for checking my results for the beam-shape coefficients obtained by numerical integration of Eqs. (5). I also thank El-

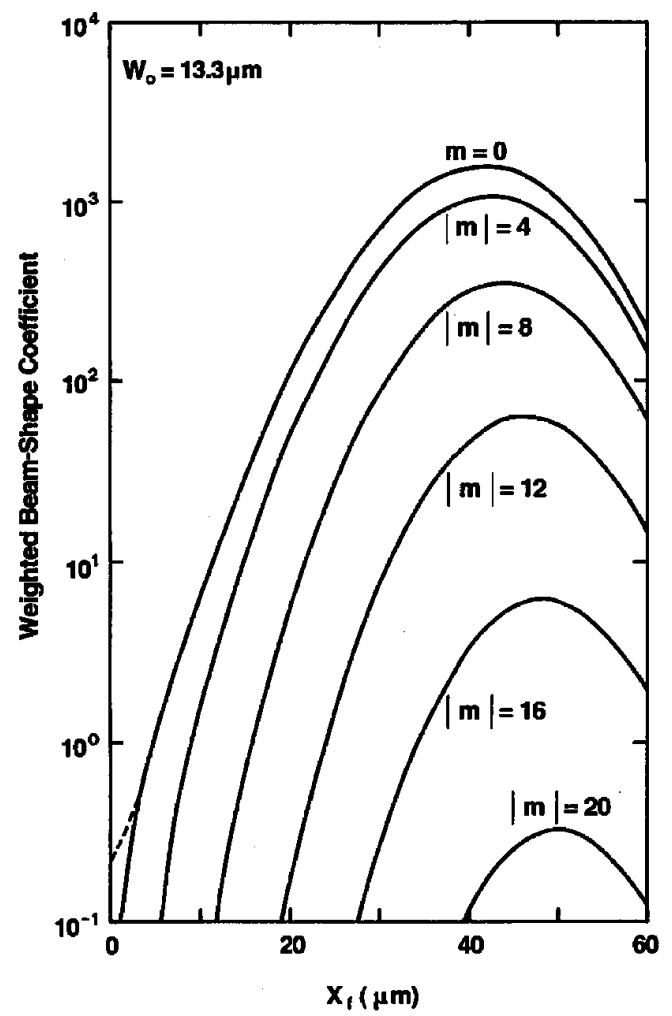

Fig. 5. Weighted beam-shape coefficient $\left|A_{I m}{ }^{l o c_{T l}} \mathrm{~m}\right|$ as a function of $x_{f}$ for $I=430$ and an off-axis Gaussian beam with $\lambda=0.6328 \mu \mathrm{m}$ and $y_{f}=z_{f}=0$. The short dashed curve is the weighted beam-shape coefficient for $|\mathrm{m}|=1$. This is the only coefficient that remains nonzero in the on-axis limit and is proportional to the MDR excitation rate by an incident plane wave. 
sayed E. M. Khalid of Assuit University, Egypt, and formerly of Clarkson University, for generously sending me his computer code for the angular spectrum of the plane waves-T-matrix scattering calculation. Last, I thank Gérard Gréhan of the Laboratoire d'Energétique des Systèmes et Procédés, Institut National des Sciences Appliquées de Rouen, France, for generously sending methe Rouen local ized approximation Gaussian beam-scattering computer code.

\section{References}

1. Lord Rayleigh, "The incidence of light upon a transparent sphere of dimensions comparable with the wave length, "Proc. R. Soc. London Ser. A 84, 25-46 (1910); Scientific Papers by Lord Rayleigh, J . N. Howard, ed. (Dover, New York, 1964), Vol. 5, paper 344, pp. 547-568.

2. J . V. Dave, "Scattering of visible light by large water spheres," Appl. Opt. 8, 155-164(1969).

3. W. J . Wiscombe, "I mproved Mie scattering al gorithms," Appl. Opt. 19, 1505-1509(1980).

4. C. F. Bohren and D. R. Huffman, Absorption and Scattering of Light by Small Particles(Wiley, New York, 1983), AppendixA.

5. P. W. Barber, D.-S. Y. Wang, and M. B. Long, "Scattering calculations using a microcomputer,"Appl. Opt. 20, 1121-1123 (1981).

6. G. Gouesbet, G. Gréhan, and B. Maheu, "Scattering of a Gaussian beam by a Mie scatter center using a Bromwich formalism,"J . Opt. (Paris) 16, 83-93 (1985),

7. G. Gouesbet, B. Maheu, and G. Gréhan, "Light scattering from a sphere arbitrarily located in a Gaussian beam, using a Bromwich formulation,"J . Opt. Soc. Am. A 5, 1427-1443 (1988).

8. J . P. Barton, D. R. Alexander, and S. A. Schaub, "Internal and near-surface electromagnetic fields for a spherical particle irradiated by a focused laser beam,"J . Appl. Phys. 64, 16321639 (1988).

9 C. Yeh, S. Colak, and P. Barber, "Scattering of sharply focused beams by arbitrarily shaped dielectric particles: an exact solution,"Appl. Opt. 21, 4426-4433 (1982).

10. E. E. M. Khaled, S. C. Hill, P. W. Barber, and D. Q. Chowdhury, "Near-resonance excitation of dielectric spheres with plane waves and off-axis Gaussian beams,"Appl. Opt. 31, 1166-1169 (1992).

11. E. E. M. Khaled, S. C. Hill, and P. W. Barber, "Scattered and internal intensity of a sphere illuminated with a Gaussian beam,"I EEE Trans. Antennas Propag. 41, 295-303 (1993).

12. L. W. Davis, "Theory of electromagnetic beams," Phys. Rev. A 19, 1177-1179 (1979).

13. J. P. Barton and D. R. Alexander, "Fifth-order corrected el ectromagnetic field components for a fundamental Gaussian beam,"J . Appl. Phys. 66, 2800-2802 (1989).

14. J. A. Lock and G. Gouesbet, "Rigorous justification of the localized approximation to the beam-shape coefficients in generalized Lorenz-Mie theory. I: On-axis beams," J . Opt. Soc. Am. A 11, 2503-2515 (1994).

15. G. Gouesbet and J. A. Lock, "Rigorous justification of the localized approximation to the beam-shape coefficients in generalized Lorenz-Mie theory. II: Off-axis beams,"J . Opt. Soc. Am. A 11, 2516-2525 (1994).

16. G. Gréhan, B. Maheu, and G. Gouesbet, "Scattering of laser beams by Mie scatter centers: numerical results using a localized approximation,"Appl. Opt. 25, 3539-3548 (1986).

17. G. Gouesbet, G. Gréhan, and B. Maheu, “Localized interpretation to compute all the coefficients $g_{n}{ }^{m}$ in the generalized Lorenz-Mietheory,"J . Opt. Soc. Am. A 7, 998-1007 (1990).

18. J . A. Lock, "Contribution of high-order rainbows to the scattering of a Gaussian laser beam by a spherical particle," J . Opt. Soc. Am. A 10, 693-706 (1993).
19. K. F. Ren, G. Gréhan, and G. Gouesbet, "Localized approximation of generalized Lorenz-Mie theory: faster algorithm for computations of beam shape coefficients, $\mathrm{g}_{\mathrm{n}}{ }^{\mathrm{m}}$," Part. Part. Syst. Charact. 9, 144-150 (1992).

20. G. Arfken, Mathematical Methods for Physicists, 3rd ed. (Academic, New York, 1985), Eq. (11.5).

21. Ref. 20, Eq. (11.114).

22. Ref. 20, Table 11.2

23. G. B. Thomas, Calculus and Analytic Geometry, 3rd ed. (Addison-Wesley, Reading, Mass., 1964), Section 16-9.

24. M. Abramowitz and I. A. Stegun, Handbook of Mathematical Functions (National Bureau of Standards, Washington, D.C., 1964), Tables 9.8-9.11.

25. Ref. 24, Eq. (9.6.32).

26. Ref. 20, Eqs. (11.129) and (11.133).

27. Ref. 24, Tables 9.1-9.4.

28. Ref. 24, Eq. (9.1.40).

29. P. W. Barber and S. C. Hill, Light Scattering by Particles: Computational Methods (World Scientific, Singapore, 1990), p. 155.

30. F. Slimani, G. Gréhan, G. Gouesbet, and D. Allano, "Near-field Lorenz-Mie theory and its application to microholography," Appl. Opt. 23, 4140-4148 (1984).

31. F. Guilloteau, G. Gréhan, and G. Gouesbet, “Optical levitation experiments to assess the validity of the generalized LorenzMietheory,"Appl. Opt. 31, 2942-2951 (1992).

32. J . A. Lock and E. A. Hovenac, "Diffraction of a Gaussian beam by a spherical obstacle,"Am. J . Phys. 61, 698-707 (1993).

33. J. P. Barton, D. R. Alexander, and S. A. Schaub, "I nternal fields of a spherical particle illuminated by a tightly focused laser beam: focal point positioning effects at resonance,"J . Appl. Phys. 65, 2900-2906 (1989).

34. J . P. Barton, D. R. Alexander, and S. A. Schaub, "Theoretical determination of net radiation force and torque for a spherical particle illuminated by a focused laser beam,"J . Appl. Phys. 66, 4594-4602 (1989).

35. E. E. M. Khaled, S. C. Hill, and P. W. Barber, "I nternal electric energy in a spherical particleilluminated with a plane wave or off-axis Gaussian beam,"Appl. Opt. 33, 524-532 (1994).

36. E. A. Hovenac and J. A. Lock, "Calibration of the forwardscattering spectrometer probe: modeling scattering from a multimode laser beam," J . Atmos. Oceanic Technol. 10, 518525 (1993).

37. S. A. Schaub, Mountain Technical Center, Schuller International, Littleton, Col. 80127 (personal communication, March 1992).

38. T. Bear, "Continuous-wave laser oscillation in a Nd:YAG sphere," Opt. Lett. 12, 392-394 (1987).

39. J .-Z. Zhang, D. H. Leach, and R. K. Chang, "Photon lifetime within a droplet: temporal determination of elastic and stimulated Raman scattering," Opt. Lett. 13, 270-272 (1988).

40. J .-Z. Zhang, G. Chen, and R. K. Chang, "Pumping of stimulated Raman scattering by stimulated Brillouin scattering within a single liquid droplet: input laser line width effects," J . Opt. Soc. Am. B 7, 108-115 (1990).

41. A. Messiah, Quantum Mechanics (Wiley, New York, 1968), Vol. 1, appendix B.II.6.

42. H. C. van de Hulst, Light Scattering by Small Particles(Dover, New York, 1981), Sect. 12.31

43. B. Maheu, G. Gréhan, and G. Gouesbet, "Ray localization in Gaussian beams," Opt. Commun. 70, 259-262 (1989).

44. C. C. Lam, P. T. Leung, and K. Young, "Explicit asymptotic formulas for the positions, widths, and strengths of resonances in Mie scattering,"J . Opt. Soc. Am. B 9, 1585-1592 (1992).

45. S. Schiller, "Asymptotic expansion of morphological resonance frequencies in Mie scattering," Appl. Opt. 32, 2181-2185 (1993).

46. Ref. 24, Sect. 10.4 . 